\title{
Sociocultural Barriers to Early Diagnosis of Autism Spectrum Disorder
}

Anum Farooq ${ }^{1}$, Shoaib Ahmed ${ }^{2}$

\section{ABSTRACT}

Objective: To qualitatively explore community, family, and health care system barriers to early diagnosis of autism spectrum disorder (ASD) in children.

Study Design: A phenomenological qualitative approach was used to explore mothers' experiences of receiving ASD diagnosis for their children.

Place and Duration of Study: The study was conducted at Autism Resource Centre Islamabad, from March 2018 to August 2018.

Materials and Methods: In-depth qualitative interviews of eight mothers of children diagnosed with ASD, who were above 36 months of age.

Results: Using thematic analysis, three major themes were identified as barriers in reaching ASD diagnosis i.e. parent's lack of knowledge and misperceptions of ASD; health care system issues like undefined pathway to care, dissatisfaction with medical or associated professionals, delays due to structural and process barriers; and family factors such as stigma around mental health and disability, family denial and financial constraints.

Conclusion: Additional educational outreach to families, de-stigmatization of ASD, streamlining the ASD diagnostic process, and providing additional support to parents of at-risk children may decrease delays in ASD diagnosis among children.

\section{Key Words: Autism Spectrum Disorder, Early Diagnosis, Sociocultural Barriers.}

How to cite this: Farooq A, Ahmed S. Sociocultural Barriers to Early Diagnosis of Autism Spectrum Disorder. Life and Science. 2020; 1(4): 139-144. doi: http://doi.org/10.37185/LnS.1.1.106

This is an Open Access article distributed under the terms of the Creative Commons Attribution License (http://creativecommons.org/licenses/by/4.0), which permits unrestricted use, distribution, and reproduction in any medium, provided the original work is properly cited.

\section{Introduction}

Autism Spectrum Disorder (ASD) is a universal neurodevelopmental disorder that persists over the lifetime. ${ }^{1}$ Literature suggests that early diagnosis will result in better prognosis and improved long term developmental and family outcomes. ${ }^{2}$ Despite its significance, most of children are diagnosed after the first three developmental years or misdiagnosed with other developmental disabilities that might hinder the prognosis. ${ }^{3}$

It is believed that the most critically transitional point

${ }^{1}$ Department of Clinical Psychology

Shifa Tameer-e-Millat University, Islamabad

${ }^{2}$ Department of Paediatrics

Hearts International Hospital, Rawalpindi

Correspondence:

Ms. Anum Farooq

Department of Clinical Psychology

Shifa Tameer-e-Millat University, Islamabad

E-mail: anum.dcp@stmu.edu.pk

Funding Source: NIL; Conflict of Interest: NIL

Received: Mar 17, 2020; Revised: Jun 25, 2020

Accepted: Sep 08, 2020

139 for parents of children with a disability, is receiving the diagnosis. ${ }^{4,5}$ However, there has been sparsity of research that examines the diagnostic process comprehensively. ${ }^{6}$ Available studies provide a preliminary picture of the diagnostic experience of children with ASD and their parents as they progress through the pre-diagnostic, diagnostic and postdiagnostic phases. ${ }^{7,8}$

There are a number of factors that possibly exacerbate the delay in diagnosis. During the prediagnostic stages, parents report ambiguity and confusion regarding the severity of the developmental delays ${ }^{9}$, lack of knowledge about developmental milestones and about red flags of autism. ${ }^{10,11}$ However, one of the major developmental concern is the language and communication barrier in their children. ${ }^{12}$ Among the other reported factors are the limited knowledge of physicians regarding the presentation, prognosis, and treatment of ASD. ${ }^{13}$ This often causes delay for an extended period of time, before the families of 
children with ASD are referred to specialists for assessment. ${ }^{14}$ Other factors include limited number of school-based health clinics and paediatricians, ${ }^{15}$ healthcare professionals' negligence or structural barriers for example limited health system resources. ${ }^{16}$ State provided services for autism in both rural and urban areas in Pakistan are almost non-existent. ${ }^{11}$ Inadequate information about the prevalence of autism, unestablished health pathways and magnitude of unmet needs are major barriers in providing services for ASD children and their families in Pakistan. Cultural and societal issues also lead to delayed diagnosis as literature has shown a lack of parents' knowledge about developmental delays and autism ${ }^{17}$, health literacy and acculturation, ${ }^{10}$ family denial of any disability, ${ }^{18,19}$ stigma attached and socially unacceptability of mental disorders. ${ }^{17}$ In the Pakistani culture, families commonly consult traditional faith healers and religious scholars to seek cure and explanation for their children's disabilities. ${ }^{11,17}$ During the post diagnostic stage, the sole responsibility of day to day care of an ASD child is almost entirely on the mother ${ }^{11}$ and the parents who struggle in finding an appropriate management and special need school for their child. ${ }^{7}$

There is a need to understand the diagnostic process, its implication and the factors that are responsible in impacting parental experiences of obtaining a diagnosis of ASD. The current study aims to explore sociodemographic barrier to early diagnosis of ASD diagnosis.

\section{Materials and Methods \\ Study Design}

A qualitative study using in-depth interviews of eight mothers.

\section{Recruitment and Eligibility}

Mothers of eight children diagnosed with autism, crosschecked on Diagnostic and Statistical Manual-5 (DSM-5) by a certified autism specialist, were interviewed.

Participants were recruited through purposive sampling from the Autism Resource Centre, Islamabad using a maximum variation sample to capture the diversity of sociodemographic characteristics.

\section{Procedure}

In-depth interviews were conducted by a bilingual researcher. Verbal informed consent was obtained prior to interviews. Demographic characteristics of the participants were collected initially, followed by an in-depth interview. Interviews were based on an interview guide having questions related to the mother's experiences of getting the diagnosis and the possible barriers that delayed the diagnosis. The facilitator asked probing and clarifying questions or asked participants to elaborate on emerging themes. The duration of the interviews was between 45-60 min, and they were conducted at Autism Resource Center Islamabad from March 2018 to August 2018. Verbatim notes were taken during the interview. Data was collected till thematic saturation was achieved.

\section{Participants}

The study involved eight mothers, aged $24-35$ years, whose children were 3-10 years old. The mothers approached were new referrals to the Autism Centre, where their children had been diagnosed with ASD by an autism specialist using DSM-5 diagnostic criteria. The diagnoses of their children were as follows: ASD requiring support (30\%), ASD requiring substantial support (50\%), and ASD requiring very substantial support (20\%). Six of the children were males and two were females. Most of participants were taking either full time or private behaviour therapy sessions in the autism institute. With respect to ethnicity, $87 \%$ of participants were Punjabi and 13\% were Pashtun. Urdu was the first language for $87 \%$ of the participants and their children.

\section{Results}

\section{Qualitative Analysis}

A total of eight 8 in-depth qualitative interviews were conducted with mothers of ASD cases diagnosed after the age of 36 months. Data was analysed using thematic analysis based on the framework analysis approach (Ritchie \& Spencer, 1994) involving five stages of analysis: familiarization with the data, identification of a thematic framework, indexing (coding raw data), charting, and finally interpretation. Interviews were audio recorded and transcribed verbatim in their original language, followed by reading and rereading for familiarization with the raw data. Coding was done manually during the data familiarization stage. Emerging codes were organized into sub-themes and themes. 
The researcher identified themes describing barriers/delay in ASD diagnosis and organized them into three major themes: Knowledge and perceptions of ASD, health care system factors, and family factors. Figure 1 showing all major themes and sub-themes.

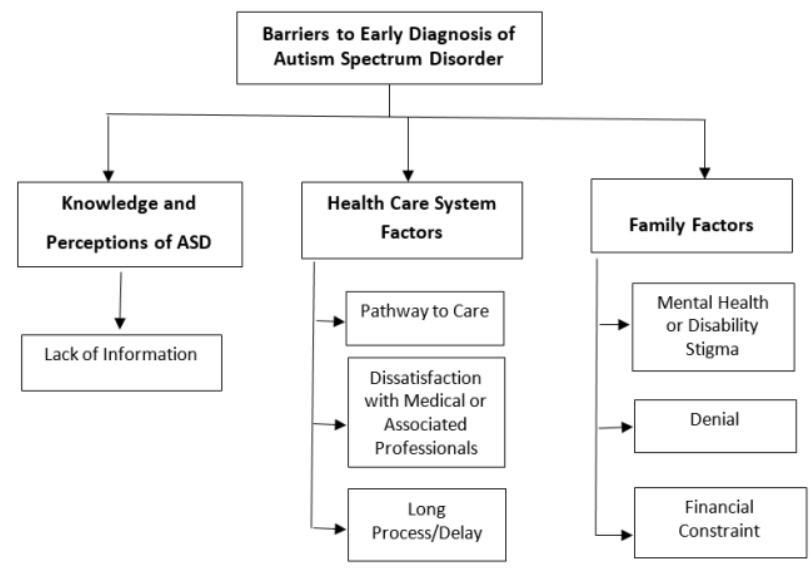

Fig 1: Major themes and sub-themes

\section{Knowledge and Perceptions of ASD}

Almost all of the parents believed that they lack the basic knowledge about ASD symptoms and were not aware about the developmental delays resulting in delayed diagnosis and seeking interventions.

A mother reported that "Our community as a whole does not have any idea about autism, its symptoms and red flags. My family was the first one to observe his unusual behaviour, but they thought it's because I neglect him due to my job."

The family and parental lack of awareness about developmental milestones and disabilities are due to the beliefs regarding such delays. They reported that they believed most of the developmental delays were due to unexplained reasons like speech delay is considered normal till age 5 years in our families, however a minor surgical procedure like tongue tie removal is usually done. A mother narrated that:

"We as parents have no basic knowledge about developmental milestones. It is something so common in our culture that we rely on the expert opinion and knowledge we get from elder family members to guide us for our children. In my case, I believe that my inability to identify his developmental delay especially speech delay, resulted into delayed diagnosis."

"My mother in law said that speech delay till the age of 3 is not a big issue, which delayed the diagnostic process."
While some parents were able to identify problems in communication and social reciprocation as core problems, they could not connect this to the behavioural problems:

"We know that he is different from my other two children, as he usually does not communicate his needs and has no words till the age of 4 years. I feel so helpless at times how to communicate or ask him about his needs."

Most of the times, parents and other family members perceive ASD as intellectual disability, rather than a developmental disorder and they believe that there is no solution to help such children except to deal them at home. A mother reported that there was a delay in seeking any diagnostic help as her in-laws endorsed that her son has intellectual disability and there is no point to waste time on diagnosis.

"According to my sister in law, he is suffering from intellectual disability and the only thing we can do is to fulfil his self-care needs, for instance nutrition and hygiene. Also, we can hire a maid who can look after and spend whole day playing with him."

\section{Health Care System Factors}

Following themes were identified as health care system factors:

1. Pathway to care: In most cases, paediatricians were the first person to consult with regard to the child's problem. However, the inability to diagnose or detect the problem by the paediatricians resulted in random visits to medical specialists. In most of the cases, the lapsed period between the first concern and diagnosis was quite long, resulting in seeking help from religious/spiritual healers or homeopathic doctors. Unclear referral pathways resulted in visits to multiple professionals (paediatric, psychiatry) hence, parents often had to instigate referrals themselves and navigate the process alone. A mother stated that:

"Both the family and doctors misguide you. They started giving examples of children who spoke at the age of 3 years or so."

Visiting multiple professionals was reported in these words by the respondents:

"My child's paediatrician was unable to identify any delays in him till age of 2 years."

"We visited multiple doctors in various cities so that 
our child gets any diagnosis, but unfortunately they failed to identify his problem. So, I took him to a spiritual healer and his behavioural difficulties are getting better."

2. Dissatisfaction with medical associated professionals: Most of the mothers reported frequent switching of health care providers before finding the one who took their concerns seriously. While sharing their experiences related to the general practitioners/ paediatricians, mothers reported that the lack of basic knowledge about developmental disabilities became a reason of misdiagnosis. Moreover, incompetence exhibited by the psychologists and speech therapists in identifying the signs and symptoms of autism resulted in visiting multiple professionals and places for diagnosis. A mother reported her experience as:

"We went to a psychologist in Peshawar, after observing my son, she was convinced that our child has no developmental disorder and has situational hyperactivity due to excessive gadget use and sugary items intake. She insisted that we should not seek any further assessment procedures and it will take a year or two till he fully recovers."

3. Long process and delays: Even after the developmental issue has been identified, the long process/delays in initiation of intervention due to structural and process barriers, further complicates and prolongs the diagnostic process. Structural barriers were defined in terms of lack of experts/professionals like autism specialists, behaviour analysts or occupational therapists in the health care departments to identify and manage ASD. Unavailability of such professionals in the paediatrics departments of every hospital makes screening and diagnostic process difficult.

Mothers reported frustration as the process to get diagnosed stretched out and often entaiedl visits to multiple medical and school personnel. Some of them became skeptical as to whether the service providers were acting in their child's best interest. As the providers' diagnoses and recommendations seemed less trustworthy, some parents no longer continue to act on their recommendations.

\section{Family Factors}

The biggest barrier faced by families in the way of getting diagnosis is the fear of mental health and disability stigma. It prevents parents from seeking professional help, getting involved socially, or pursuingtheir careers. A mother narrated:

"My socialization is limited after his diagnosis. His grandmother used to send him at my parent's place when we had any gathering so that people don't know about my son and his disability."

Similarly related to pursuing one's career, a mother reported,

"I am an engineer, but I discontinued my job due to his disability as no one else in the family was ready to help me".

"People label such children as abnormal (pagal), they avoid such children and feel pity for parents. I do not find people welcoming for autistic children in our society, so I have to decide in which gathering I have to take my son".

Most of the mothers reported that they served as the primary carer for their child, and they are considered responsible for every need of their child. A few reported that they hired domestic servants to assist them with childcare. Despite being the primarycarer, they are being blamed for their child's disability. This results in marital discord and difficult interpersonal relationship among families. A mother described her experience as:

"I was in severe depression during the time he was not getting diagnosis as my in-laws used to blame me for his disability. This resulted in frequent fights and ended up in marital discord."

"My in-laws did not accept him with his disability so I was forced to move to my mother's home with him. Though we ended up in divorce 5 years after his birth."

Denial in the families related to the child's disability and to seek professional help and under reporting of the symptoms are some of the factors identified by the parents. A mother reported her denial in these words:

"I admit that the fear of him, having a diagnosis, was so unbearable that I used to tell major symptoms to the doctors only and denied that he is having other behavioural issues."

Parents defined financial constraints in terms of travelling and accommodation expenses for seeking expert opinion/follow-up sessions as all the services related to developmental disabilities are available in big cities. These issues were reported in these words: 
"The issue in small cities is that they have no services and parents have to rush to the big cities. We moved from Peshawar just to get his diagnosis and some sort of treatment."

\section{Discussion}

The current study provides a comprehensive overview of the mothers' experiences in getting the diagnosis for their children. Three major categories were identified as barriers in getting ASD diagnosis i.e. Knowledge and perceptions of ASD, Health care system and family factors. Parents lack the basic knowledge about ASD symptoms. There is also a lack of awareness regarding the developmental milestones which leads to delayed identification of such problems. The literature aligns with the lack of awareness and insufficient knowledge about autism among parents especially prior to their child's diagnosis ${ }^{7,20}$ and this lack of knowledge impedes early ASD identification. ${ }^{16}$

Another theme highlighted by the current study is the inefficient health care system that resulted in delayed diagnosis due to the lengthy process of being referred to the concerned specialist and getting the diagnosis. These issues were also reported in the Latino community (Portland, USA) where the diagnosis was delayed due to long processes. ${ }^{16}$ The unidentified pathways to ASD care were the results of unclear diagnostic services, untrained professionals to identify ASD red flags ${ }^{21,22}$ and inability to make appropriate referrals. ${ }^{7,23}$

Moreover, the mothers reported their concern that a few good services that are available are concentrated in the urban areas and inaccessible to the majority. ${ }^{11}$ However, the qualitative exploration showed an alternative practice, parents used based on their cultural preferences. These includes homeopathy and visits to religious and spiritual healers. ${ }^{11,17}$

Mental health and disability stigma are the greatest concerns of the Pakistani society. ${ }^{11}$ Health professionals and parents at times are reluctant to make diagnosis because of fear of labelling the child. ${ }^{17}$ This collective pattern prevails in the society because of the notion created in our society related to the disabilities ${ }^{24}$, families' unacceptance for such disabilities, and blaming mothers for their child's disability. ${ }^{17}$

Mothers also highlighted the financial constraints due to unavailability of autism services in small cities/villages. Approaching the professionals is a major structural barrier in getting diagnosed. The same issues were reported in a country report by Imran and Azeem (2014) which draws attention to the discrepancy in provision of mental health services in urban and rural areas of Pakistan. ${ }^{17}$ Majority of the rural population have no access to mental health care professionals or tertiary health care services. Very few trained health-care workers and special education schools exist in cities even the available health-care workers are not proficient in diagnosing and managing children with autism. ${ }^{17,25}$ The management of autism requires a highly specialized manpower and due to limited resources in developing countries, only a few professionals equipped with these evidence based interventions are present. ${ }^{26}$

All the factors identified in the current study gives a preamble of the major factors which delays the diagnosis and subsequent intervention for ASD.

\section{Conclusion}

The study highlights that the pathway to seek diagnosis for children with developmental disabilities like autism, is unclear and uncoordinated. Mothers reported significantly more negative experiences during the diagnostic process than positive. Lack of professional competency to identify/diagnose ASD, unclear referral pathways, rediagnosis from multiple institutes and professionals, lack of healthcare services for ASDs in small towns/cities, stigma, family/parents denial, are some of the factors identified by the study that delay the diagnostic process.

\section{Acknowledgements}

Prof. Dr. Syed Muhammad Imran Majeed, Dr. Arif Mahmud and Ms. Taskeen Mansoor participated in technical editing of the manuscript and proof reading. Prof. Dr. Mujtaba Quadri critically reviewed the study proposal.

\section{REFERENCES}

1. Diagnostic and Statistical Manual of Mental Disorders: DSM-5 ( $5^{\text {th }}$ edition) Washington, DC American Psychiatric Association; 2013.

2. Johnson CP, Myers SM. American Academy of Pediatrics Council on Children with Disabilities. Identification and evaluation of children with autism spectrum disorders. J Pediatr. 2007; 120: 1183-1215. 
3. Merten EC, Cwik JC, Margraf J, Schneider S. Over diagnosis of mental disorders in children and adolescents (in developed countries). Child Adolesc Psychiatry Ment Health. 2017; 11: 5.

4. Abbott M, Bernard P, Forge J. Communicating a diagnosis of autism spectrum disorder - A qualitative study of parents' experiences. Clin Child Psychol P. 2012; 18:370-82.

5. Evan A. Parents' responses to their child's diagnosis of autism spectrum disorder [unpublished dissertation] University of Southampton; 2010.

6. Braiden HJ, Bothwell J, Duffy J. Parents' experience of the diagnostic process for autistic spectrum disorders. Child Care Pract. 2010; 16: 377-89.

7. Wong V, Yu Y, Keyes ML, McGrew JH. Pre-diagnostic and diagnostic stages of Autism Spectrum Disorder: A Parent Perspective. Child Care Pract. 2017; 23: 195-217.

8. Mandell DS, Novak MM, Zubritsky, CD. Factors Associated With Age of Diagnosis Among Children With Autism Spectrum Disorders. J Pediatr. 2005; 116: 1480-6.

9. Midence $\mathrm{K}, \mathrm{O}^{\prime} \mathrm{Neill} \mathrm{M}$. The experience of parents in the diagnosis of autism: A pilot study. Autism. 1999; 3: 273-85.

10. Keels M. Ethnic group differences in early head start parents' parenting beliefs and practices and links to children's early cognitive development. Early Child. Res. Q. 2009; 24: 381-97.

11. Minhas A, Vajaratkar V, Divan G, Hamdani SU, Leadbitter K, Taylor C, et al. Parents' perspectives on care of children with autistic spectrum disorder in South Asia - Views from Pakistan and India. Int Rev Psychiatry. 2015; 27: 247-56.

12. Kozlowski AM, Matson JL, Horovitz M, Worley JA, Neal D. Parents' first concerns of their child's development in toddlers with autism spectrum disorders. Dev Neurorehabil. 2011; 14: 72-8.

13. Shah K. What Do Medical Students Know about Autism? Autism. 2001; 5: 127-33.

14. Baird G, Cass H, Slonims V. Diagnosis of Autism. Br. Med. J. 2003; 327: 488-93.

15. Mandell D, Palmer R. Differences Among States in the Identification of Autistic Spectrum Disorders. Arch Pediatr Adolesc Med. 2005; 159: 266-9.

16. Zuckerman K, Sinche B, Mejia A, Cobian M, Becker T, Nicolaidis C. Latino Parents' Perspectives on Barriers to
Autism Diagnosis. Acad Pediatr. 2014; 14: 301-8.

17. Imran N, Azeem MW. Autism Spectrum Disorder; perspective from Pakistan. In comprehensive guide to Autism. Springer New York. 2014; pp: 2483-96.

18. Mandell D, Listerud J, Levy S, Pinto-Martin J. Race Differences in the Age at Diagnosis Among MedicaidEligible Children with Autism. J AM Acad Child Psy. 2002; 41: 1447-53.

19. Mandell D, Ittenbach R, Levy S, Pinto-Martin J. Disparities in Diagnoses Received Prior to a Diagnosis of Autism Spectrum Disorder. J. Autism Dev. Disord. 2006; 37: 17951802.

20. Anwar M, Tahir M, Nusrat K, Khan M. Knowledge, Awareness, and Perceptions Regarding Autism among Parents in Karachi, Pakistan. Cureus. 2018; 10: e3299.

21. Khan F, Shehzad RK, Chaudhry HR. Child and adolescent mental health services in Pakistan: Current situation, future directions and possible solutions. International Review of Psychiatry. 2008; 5: 86-88.

22. Hussein SA. A review of global issues and prevalence of child mental health Problems; Where does $\mathrm{CAMH}$ stand in Pakistan? Journal of Pakistan Psychiatric Society. 2009; 6: 513.

23. Imran N, Chaudry MR, Azeem MW, Bhatti MR, Choudhary $\mathrm{ZI}$, Cheem MA. A survey of Autism knowledge and attitudes among the healthcare professionals in Lahore, Pakistan. BMC Pediatr. 2011; 11: 107.

24. McKay MM, Pennington J, Lynn CJ, McCadam K. Understanding urban child mental health service use: Two studies of child, family, and environmental correlates. J Behav Health Ser R. 2011; 28: 475-83.

25. Imran N, Azeem MW, Sattar A, Bhatti MR. Frequency of ICD10 psychiatric diagnosis in children with intellectual disability in Lahore, Pakistan \& caregiver's perspective. Pak J Med Sci. 2015; 31, 285-9.

26. Divan G, Hamdani SU, Vajartkar V, Minhas A, Taylor C, Aldred $C$, et al. Adapting an evidence-based intervention for autism spectrum disorder for scaling up in resourceconstrained settings: the development of the PASS intervention in South Asia. Glob Health Action. 2015; 8: 27278. 